\title{
Trendbericht Physikalische Chemie
}

Kalte Moleküle

\author{
Jochen Küpper ${ }^{\Uparrow}$ and Gerard Meijer \\ Fritz-Haber-Institut der Max-Planck-Gesellschaft \\ Faradayweg 4-6, D-14195 Berlin
}

(Dated: 2. März 2008) 
Die Darstellung und Untersuchung kalter Moleküle $(\ll 1 \mathrm{~K})$ hat im letzten Jahrzehnt rasante Fortschritte gemacht. ${ }^{1]}$ Das erste Ziel dieser Experimente ist die vollständige Kontrolle aller Freiheitsgrade neutraler Moleküle. Das heißt, dass sich idealerweise alle Moleküle in einem einzigen Quantenzustand befinden und ihre Bewegung im Raum so weit eingeschränkt und kontrolliert ist, dass sie gefangen werden können. Als bisher kälteste dargestellte Molekülproben konnten Alkalidimere direkt aus ultrakalten Atomen im nano-Kelvin Bereich dargestellt werden. Einige der so erzeugten ungewöhnlichen Moleküle konnten bereits Bose-Einstein-kondensiert werden. [2]

Für chemische Fragestellungen interessanter sind direkte Methoden, die für möglichst viele verschiedenartige Moleküle anwendbar sind. Die vorhandenen Methoden nutzen z. B. die Puffergaskühlung in Heliumkryostaten oder das Selektieren, Abbremsen und Fangen von Molekülen aus Molekularstrahlen. ${ }^{1}$ Insbesondere mit der Methode der Stark-Abbremsung ${ }^{\sqrt{34}}$ wurden mittlerweile von verschiedenen Arbeitsgruppen eine Reihe kleiner Moleküle aus Überschallstrahlen abgebremst (metastabiles $\mathrm{CO}, \mathrm{NH}_{3}, \mathrm{ND}_{3}, \mathrm{OH}$, $\left.\mathrm{NH}, \mathrm{SO}_{2}, \mathrm{H}_{2} \mathrm{CO}\right)$ und elektrostatisch gefangen $\left(\mathrm{ND}_{3}, \mathrm{OH}\right.$, metastabiles $\left.\mathrm{CO}, \mathrm{NH}\right)$.

In Abb. 1 ist dargestellt, wie die Wechselwirkung der Moleküle mit elektrischen Feldern (Stark-Effekt) zu einer Ab- oder Zunahme der potentiellen Energie führt, abhängig vom Quantenzustand des Moleküls. Bei der Stark-Abbremsung von Molekülen wird die aus der Änderung der potentiellen Energie in inhomogenen elektrischen Feldern resultierende Kraft genutzt, um die Moleküle schrittweise abzubremsen. In einem einzelnen Abbremsschritt, wie er in Abb. 1 gezeigt ist, können typischerweise einige Wellenzahlen $\left(\mathrm{cm}^{-1}\right)$ kinetische Energie, etwa $1 \%$ der Anfangsenergie, entfernt werden. Wird der Prozess in einem Abbremser ca. einhundert Mal wiederholt, können die Moleküle bis zum Stillstand abgebremst werden. Der Aufbau eines solchen Experiments ist in Abb. 2 illustriert. Diese langsamen Moleküle können mit einem letzten Potentialhügel, den sie nicht mehr überwinden können, in der Mitte einer elektrostatischen Falle gestoppt und anschließend im Minimum des elektrischen Feldes gefangen werden. So können Moleküle auf beliebige Geschwindigkeiten abgebremst werden ${ }^{3}$ und für Sekunden in einer Falle gefangen werden. ${ }^{5}$ Da der Stark-Effekt eine Eigenschaft des jeweiligen Quantenzustands ist, wird bei dem Prozess prinzipiell ein einzelner Quantenzustand selektiert. 


\section{Experimente mit kalten Molekülen}

Führt man unter identischen experimentellen Bedingungen die Bestimmung der Fallenlebensdauer mit $\mathrm{OH}$-Radikalen im Schwingungsgrundzustandes $(v=0, J=3 / 2)$ und im ersten angeregten Schwingungszustandes $(v=1, J=3 / 2)$ durch, so kann man aus den in Abb. 3 gezeigten Abklingkurven direkt die Lebensdauer dieses angeregten Schwingungszustandes zu 59.0 ms bestimmen.

Weiterhin kann die Verlangsamung der Moleküle auch zur Erhöhung der Auflösung spektroskopischer Messungen dienen. In Abb. 4 ist dargestellt, wie die resultierende längere Wechselwirkungszeit des Moleküls mit dem elektromagnetischen Feld genutzt wurde, um die erreichbare Auflösung zu erhöhen. Dadurch konnte erstmals die Hyperfeinstruktur des Inversionsübergangs von ${ }^{15} \mathrm{ND}_{3}$ aufgelöst werden. ${ }^{7}$ Solche hochpräzisen Messungen können z. B. bei der Suche nach der Paritätsverletzung in chiralen Molekülen ${ }^{899}$ oder der Änderung von fundamentalen Konstanten mit der Zeit helfen. ${ }^{10}$

Die Möglichkeit, Pakete von Molekülen in einem einzigen Quantenzustand und mit beliebiger, wohldefinierter Geschwindigkeit zu erzeugen, erlaubt auch extrem detaillierte Untersuchungen von elementaren Reaktionen. In einer ersten Untersuchung zur Streuung von so kontrollierten $\mathrm{OH}$-Radikalen an Xe wurden die Streuquerschnitte verschiedener inelastischer Prozesse, die in Abb. 5 angegeben sind, ermittelt und die Prozesse im Bereich der Energieschwellen sehr genau untersucht. 11

\section{Kalte große Moleküle}

In unserer Arbeitsgruppe wurde die Stark-Abbremsung neutraler, polarer Moleküle in den letzten Jahren so erweitert, $\frac{12}{1}$ dass auch große Moleküle abgebremst werden können.13]14 Diese Techniken erlauben die räumliche Trennung einzelner Konformere, was wir für cis- und trans-3-Aminophenol (Abb. 6) bereits experimentell demonstriert haben. ${ }^{1415}$ Diese Konformerentrennung kann mittels des in Abb. 7 abgebildeten $1 \mathrm{~m}$ langen $m / \mu$-Filters erreicht werden, der äquivalent zu den $m / q$-Massenfiltern für geladene Teilchen funktioniert. Diese reinen Proben großer Moleküle sind ein wichtiger Schritt in ihrer detaillierten Untersuchung. Sie unterstützen die spektroskopische Strukturaufklärung $\sqrt{16}$ und erlauben neuartige Experimente zur Untersuchung der fs- 
Dynamik einzelner Spezies, z. B. von Biomolekülen, und der direkten Strukturbestimmung in Beugungsexperimenten an orientierten individuellen Konformeren.

Insgesamt bieten die hier vorgestellten Methoden zur Kontrolle von Molekülen faszinierende Möglichkeiten von der präzisen Spektroskopie über Reaktionsdynamikuntersuchungen bis zur ultrakalten Chemie, in der Reaktionen durch Tunnelprozesse dominiert sind.

* jochen@fhi-berlin.mpg.de

1 Special Issue “Ultracold Polar Molecules: Formation and Collisions”, Eur. Phys. J. D 31, 149 (2004).

2 J. H. Denschlag, H.-C. Nägerl, and R. Grimm, Phys. J. 3, 33 (2003).

3 H. L. Bethlem, G. Berden, and G. Meijer, Phys. Rev. Lett. 83, 1558 (1999).

4 B. van de Meerakker and G. Meijer, Phys. unserer Zeit 38, 128 (2007).

5 S. Y. T. van de Meerakker, P. H. M. Smeets, N. Vanhaecke, R. T. Jongma, and G. Meijer, Phys. Rev. Lett. 94, 023004 (2005).

6 S. Y. T. van de Meerakker, N. Vanhaecke, M. P. J. van der Loo, G. C. Groenenboom, and G. Meijer, Phys. Rev. Lett. 95, 013003 (2005).

7 J. van Veldhoven, J. Küpper, H. L. Bethlem, B. Sartakov, A. J. van Roij, and G. Meijer, Eur. Phys. J. D 31, 337 (2004).

8 C. Daussy, T. Marrel, A. Amy-Klein, C. T. Nguyen, C. J. Bordé, and C. Chardonnet, Phys. Rev. Lett. 83, 1554 (1999).

9 M. Quack and J. Stohner, Nachr. Chem. 54, 282 (2006), Trendbericht Physikalische Chemie 2005.

10 E. R. Hudson, H. J. Lewandowski, B. C. Sawyer, and J. Ye, Phys. Rev. Lett. 96, 143004 (2006).

11 J. J. Gilijamse, S. Hoekstra, S. Y. T. van de Meerakker, G. C. Groenenboom, and G. Meijer, Science 313, 1617 (2006).

12 H. L. Bethlem, M. R. Tarbutt, J. Küpper, D. Carty, K. Wohlfart, E. A. Hinds, and G. Meijer, J. Phys. B 39, R263 (2006).

13 K. Wohlfart, F. Grätz, F. Filsinger, H. Haak, G. Meijer, and J. Küpper (2007), submitted to Phys. Rev. A.

14 http://www.fhi-berlin.mpg.de/mp/jochen 

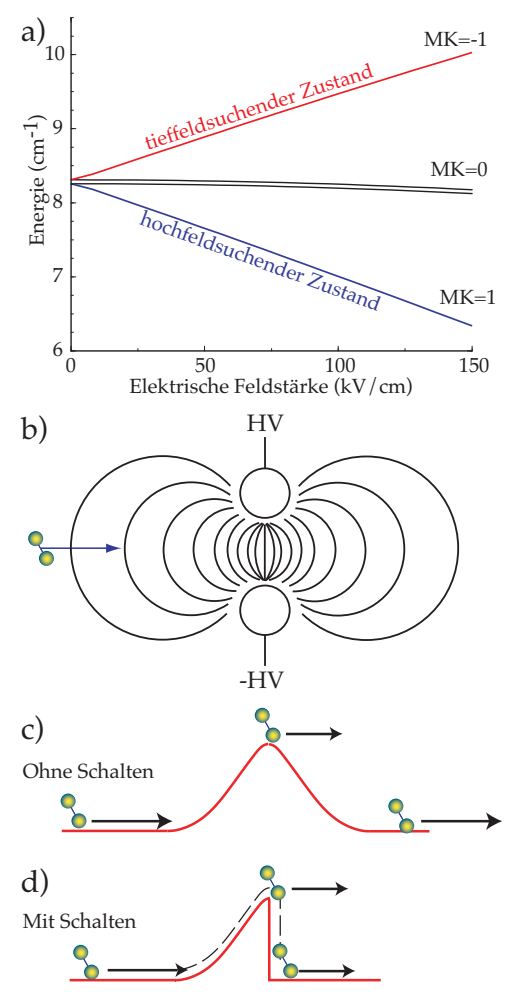

Abbildung 1: Prinzip der Stark-Abbremsung. (a) Der Stark-Effekt von $\mathrm{ND}_{3}$ im $J_{K}=1_{1}$ Rotationszustand. (b) Eine Hochspannung zwischen zwei Elektroden erzeugt ein starkes inhomogenes elektrisches Feld. (c) Auf polare Moleküle in tieffeldsuchenden Zuständen wirkt dieses elektrische Feld wie ein Potentialhügel, und bei angemessenem Schalten der Felder werden die Moleküle abgebremst. $\underline{3}$

15 F. Filsinger, U. Erlekam, G. von Helden, J. Küpper, and G. Meijer (2008), accepted for publication in Phys. Rev. Lett., preprint available at http://arxiv.org/abs/0802.2795

16 C. Riehn, Nachr. Chem. 54, 288 (2006), Trendbericht Physikalische Chemie 2005. 


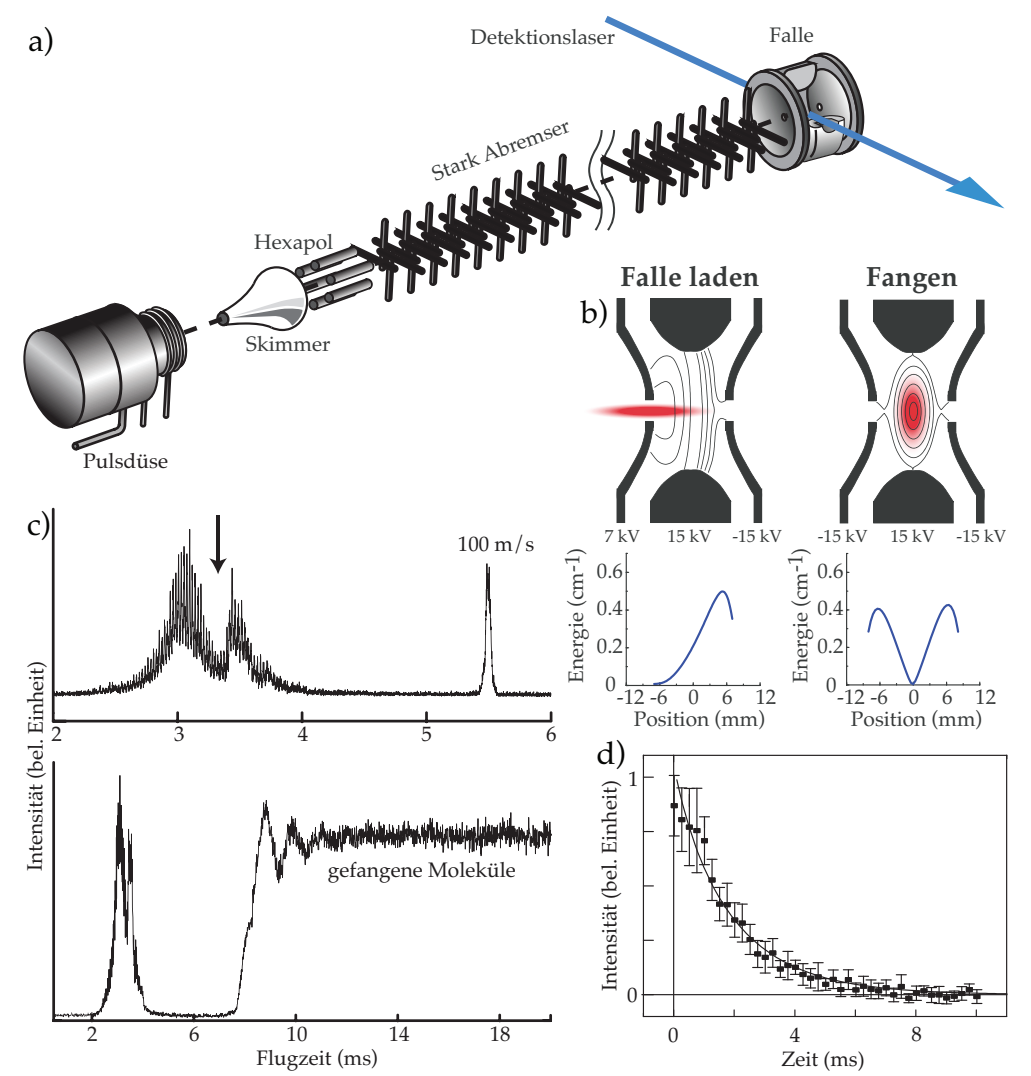

Abbildung 2: (a) Experimenteller Aufbau des Stark-Abbremsers. (b) Feldkonfigurationen zum Laden und Fangen von Molekülen in einer elektrostatischen Falle. (c) Messungen der Flugzeit von $\mathrm{OH}-$ Radikalen von der Düse zum Detektor. (d) Lebensdauer der gefangenen Moleküle ${ }^{5}$

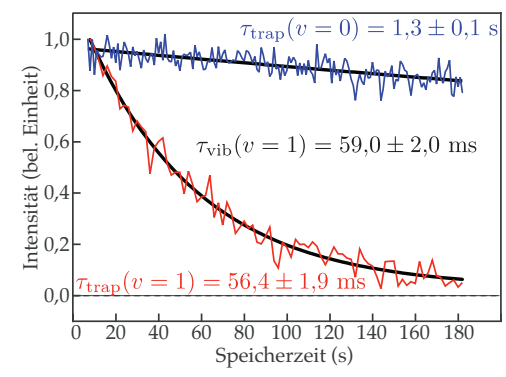

Abbildung 3: Direkte Bestimmung der Schwingungslebensdauer des ersten angeregten Schwingungszustands von $\mathrm{OH}\left({ }^{2} \Pi_{3 / 2}, v=1, J=3 / 2\right)$ aus den unterschiedlichen Fallenlebensdauern von Molekülproben im $v=0$ und $v=1$ Zustand $[6$ 


\section{Frequenz $(\mathrm{MHz})$}
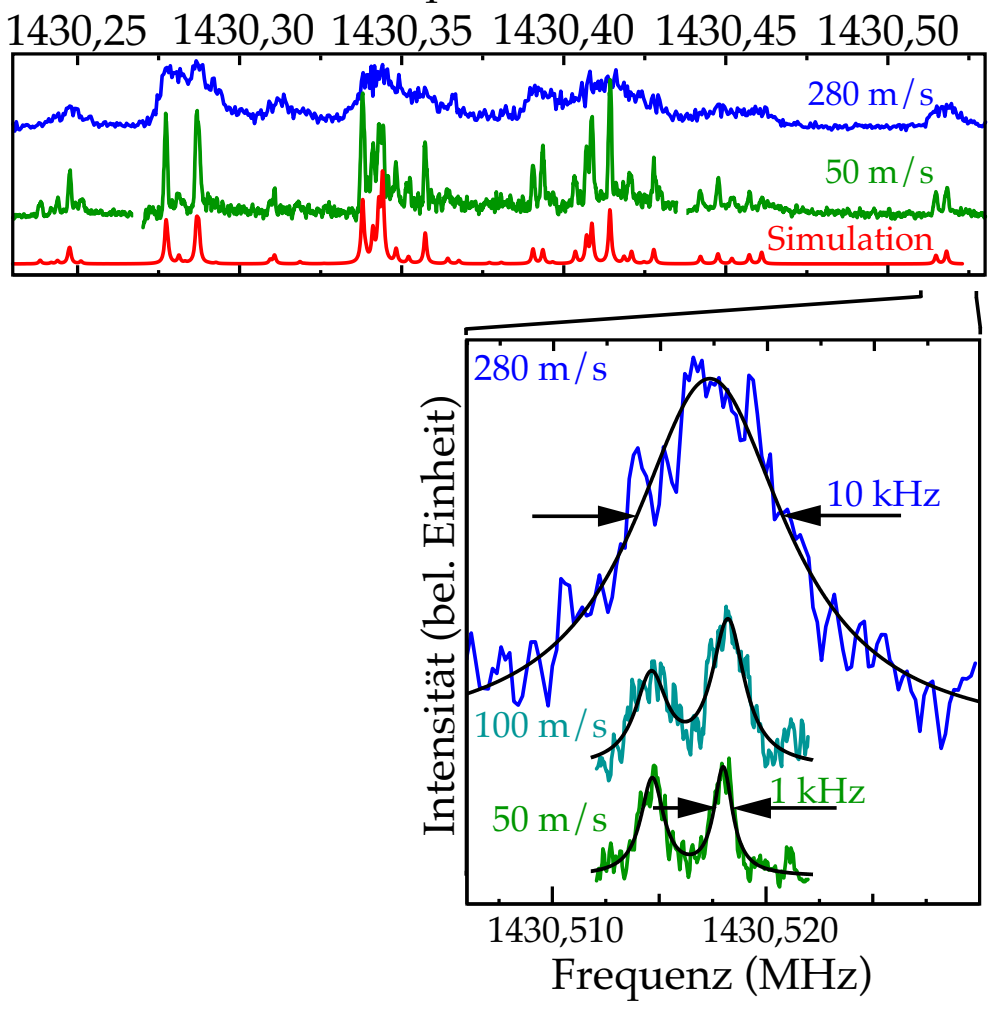

Abbildung 4: Hochaufgelöstes Spektrum des Inversionsübergangs von ${ }^{15} \mathrm{ND}_{3}$ im $J_{K}=1_{1}$ Zustand bei verschiedenen Geschwindigkeiten. Durch die Verlangsamung der Molekülpakete kann die Wechselwirkungszeit der Moleküle mit dem Mikrowellenfeld erhöht werden und die gesamte Hyperfeinstruktur des Übergangs aufgelöst werden. ${ }^{7}$
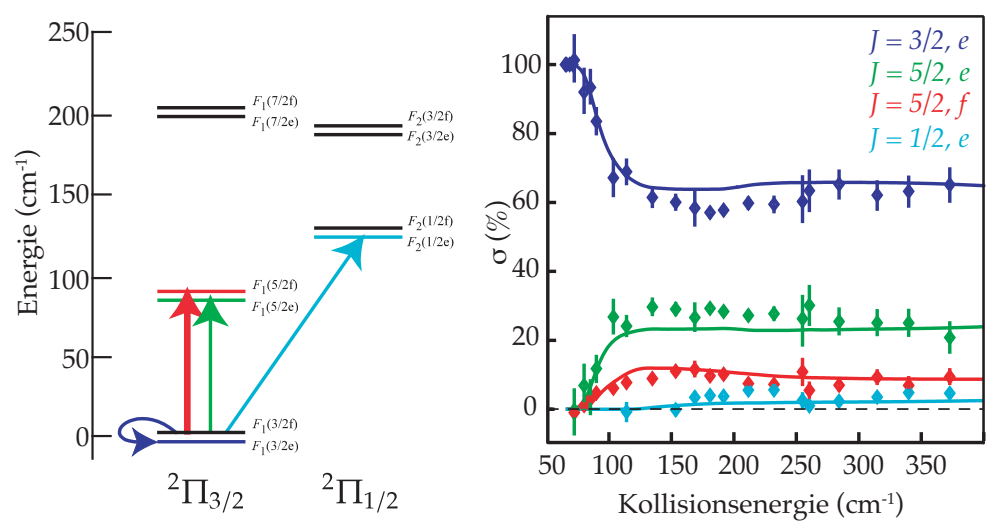

Abbildung 5: (a) Energieniveauschema der niedrigsten Zustände von $\mathrm{OH}$. Die Pfeile geben die Übergänge an, deren Streuquerschnitte experimentell untersucht wurden. (b) Abhängigkeit der relativen Streuquerschnitte von der Kollisionsenergie.11 


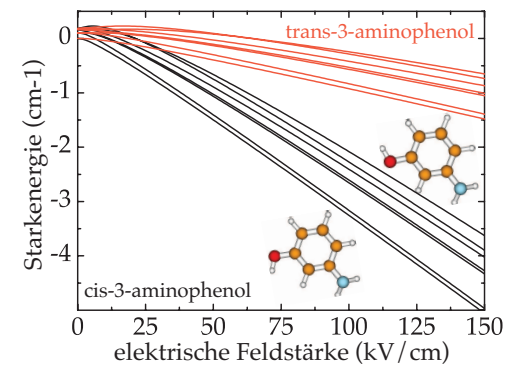

Abbildung 6: Die Molekülstrukturen von cis- und trans-3-Aminophenol mit ihren jeweiligen Dipolmomenten und den daraus berechneten Stark-Energiekurven.

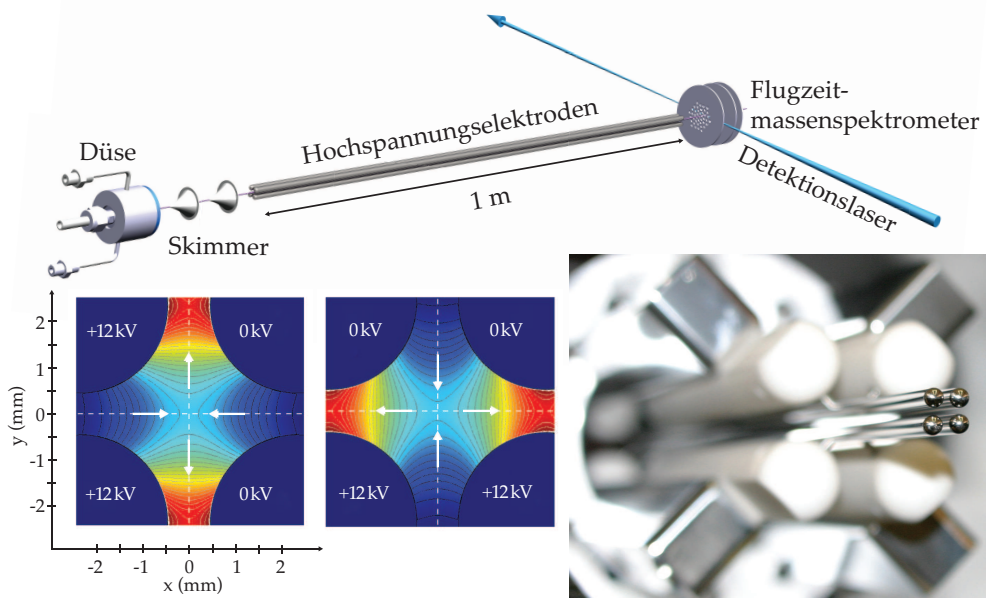

Abbildung 7: In diesem $m / \mu$-Filter können Konformere neutraler Moleküle räumlich voneinander getrennt werden, indem regelmäßig zwischen den beiden dargestellten Konfigurationen des elektrischen Feldes umgeschaltet wird. 